\title{
Functional and Structural Study of the Amino Acid Substitution in a Novel Familial Androgen Receptor Mutation (W752G) Responsible for Complete Androgen Insensitivity Syndrome
}

\author{
Françoise Paris ${ }^{a, b}, e, f \quad$ Abdelhay Boulahtouf ${ }^{d-g} \quad$ Nicolas Kalfa ${ }^{c, f} \quad$ Marie P. Guibal ${ }^{c}$ \\ Laura Gaspari $^{a}$ Nadège Servant ${ }^{b}$ William Bourguet ${ }^{f, h, i}$ Charles Sultan $^{a, b, f}$ \\ Patrick Balaguer ${ }^{\mathrm{d}-\mathrm{g}}$ \\ a Unité d'Endocrinologie et de Gynécologie Pédiatrique, Département de Pédiatrie, Hôpital Arnaud de Villeneuve, \\ and ${ }^{b}$ Département d'Hormonologie and ' Département de Chirurgie Pédiatrique, Hôpital Lapeyronie, CHU de

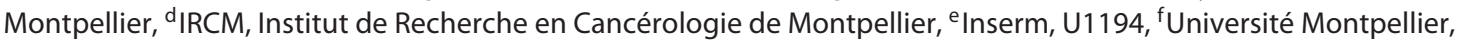 \\ gICM, Institut régional du Cancer de Montpellier, ${ }^{\mathrm{h}}$ Inserm U1054, and ${ }^{\mathrm{C} C N R S}$ UMR5048, Centre de Biochimie \\ Structurale, Montpellier, France
}

\section{Keywords}

Androgen insensitivity syndrome · Disorders of sex

development $\cdot$ Genotype/phenotype correlation and structural studies according to the nature of the amino acid substitution, which in turn may have interesting impacts on the follow-up of these patients.

(c) 2018 S. Karger AG, Basel

\begin{abstract}
Mutations of the androgen receptor $(A R)$ gene are the most frequent cause of $46, X Y$ disorders of sex development. They are associated with a variety of phenotypes, ranging from phenotypic women (complete androgen insensitivity syndrome, CAIS) to milder degrees of undervirilization (partial forms) or men with only infertility (mild form). We identified a new W752G AR mutation responsible for a familial case of CAIS and performed an in vitro study and structural analysis of this mutation and the only other reported substitution affecting the same amino acid (W752R). Although sex assignment is not discussed in cases of CAIS, we show how the phenotype-genotype correlation can be refined by in vitro
\end{abstract}

\section{KARGER}

(c) 2018 S. Karger AG, Basel

E-Mail karger@karger.com

www.karger.com/sxd
Androgen insensitivity syndrome (AIS) is the most frequent cause of disorders of sex development in patients with a 46,XY karyotype and a normal/high testosterone level (30-40\% in our experience) [Sultan et al., 2014]. Usually, it is caused due to a mutation in the androgen receptor $(A R)$ gene, which codes for the cellular mediator of androgen actions vital for masculinizing the external genitalia during fetal life and puberty. Androgen insensitivity is clinically quite heterogeneous, with the androgenic effects of testosterone (T) and dihydrotestosterone (DHT) on androgen-dependent tissues completely or partially abolished, leading respectively to complete
Prof. Françoise Paris

Unité d'Endocrinologie et de Gynécologie Pédiatrique, Département de Pédiatrie Hôpital Arnaud de Villeneuve, CHU de Montpellier

FR-34090 Montpellier (France)

E-Mail f-paris@chu-montpellier.fr 
(CAIS) or partial (PAIS) androgen insensitivity. The estimated prevalence of CAIS ranges from about $1 / 20,000$ to $1 / 64,000$ male births. The timing of referral of CAISaffected individuals to clinicians extends from birth, or even before birth, to adulthood.

Individuals with CAIS are born unambiguously female and are generally not suspected of being 46,XY until the onset of puberty. The most typical presentation is thus primary amenorrhea in a phenotypic female adolescent with normal breast development contrasting with poorly developed axillary and pubic hair. Height is typically normal to slightly increased, and the genitourinary exam reveals normal external genitalia and a rudimentary, blindending vagina. During fetal life, referral is based on a discrepancy between a 46,XY karyotype in amniocentesis and the presence of female external genitalia in prenatal ultrasound examination. In both the neonatal period and infancy, CAIS should be suspected in cases of an inguinal hernia in a phenotypic female. Recent data indicate a $1 \%$ incidence rate of CAIS in children with premenarchal inguinal hernia, and about $90 \%$ of girls with CAIS eventually develop an inguinal hernia [Sarpel et al., 2005; Hurme et al., 2009].

CAIS is related to a severe defect of AR function during fetal life that prevents normal and complete fetal virilization. The $A R$ gene is located on the $\mathrm{X}$ chromosome (Xq11q12) and comprises about $90 \mathrm{kB}$. The gene contains 8 exons and encodes a protein consisting of 919 amino acids [Eisermann et al., 2013]. The protein has 4 major functional domains: the N-terminal domain (NTD, residue 1-556) encoded by exon 1, the central DNA-binding domain (DBD, residues 557-627) encoded by exons 2 and 3, a C-terminal ligand-binding domain (LBD, residues 670-919) encoded by exons $4-8$, and a hinge region connecting the LBD to the DBD (residues 628-669) [Sultan et al., 2014].

AIS is thus transmitted in an X-linked recessive manner. To date, more than $500 A R$ gene mutations have been reported [Philibert et al., 2010; Gottlieb et al., 2012]. These mutations may be located, in decreasing frequency, in the exons encoding the LBD (exons 4-8) [Poujol et al., 2002; Georget et al., 2006], the exons of the DBD (exon 2 and 3), and exon 1 [Philibert et al., 2010].

Although newborns diagnosed as CAIS carriers are always raised as females, the time of gonadectomy tends to be later today than it was some years ago [Patel et al., 2016; Dohnert et al., 2017]. This later timing allows spontaneous puberty, but it raises the question not only of the increased testicular tumor risk [Dohnert et al., 2017] but also of both potential pubertal virilization and male iden- tity in cases of persistent residual AR activity. To better predict the future pubertal development based on the nature of the amino acid substitution, we performed an in vitro study of a new W752G mutation that we found in a newborn girl with an inguinal hernia in parallel with another reported mutation W752R [Brinkmann et al., 1995; Boehmer et al., 2001].

\section{Patient and Methods}

\section{Clinical Presentation}

The newborn girl presented at the pediatric surgery unit of the University Hospital of Montpellier for inguinal hernia at the age of 2 months. Clinical examination found 2 gonads in the inguinal position, and ultrasonography showed the presence of gonads in the hernia sac, whereas no uterus was identified. Basal plasma $\mathrm{T}$ was $0.9 \mathrm{ng} / \mathrm{mL}$ with luteinizing hormone $(\mathrm{LH})$ and follicle-stimulating hormone (FSH) levels of 0.2 and $1 \mathrm{U} / \mathrm{L}$, respectively. Antimüllerian hormone (AMH) level was $760 \mathrm{pM}$ (norm value for girls this age: $0.7-61 \mathrm{pM}$ ). Inguinal surgery confirmed the presence of gonads in both the right and left hernia sac, and the gonads were reintegrated in the abdominal position. The patient's karyotype was 46,XY. Questioning of the family revealed that one of the mother's sisters was infertile. The diagnosis of CAIS was discussed, and $A R$ gene analysis was performed.

\section{AR Gene Sequencing}

After PCR amplification of exons 1-8 of AR using the Taq PCR Master Mix kit (QIAGEN, Courtaboeuf, France), we performed direct sequencing using the BigDye terminator v1.1 kit (Applied Biosystems, Foster City, CA, USA) and an ABI Prism310 Genetic Analyzer (Applera, Courtaboeuf, France) as reported elsewhere [Philibert et al., 2010]. In case of a mutation, PCR and sequencing of the DNA sample were repeated twice to confirm the finding and rule out any PCR-generated errors. Every PCR product was sequenced with forward and reverse primers. The amino acid numbering for the AR was based on the NCBI reference sequence NM_000044.2 and the AR database [Gottlieb et al., 2012].

\section{Transient Transactivation Experiments}

AR wild type, W752G, and W752R activity was monitored on ARE(RAD9) ${ }_{6}$-collagenase-luciferase reporter constructs in U2OS cells. The AR (1-939 nt) coding sequence was inserted into the EcoRI site of the pSG5puro plasmid kindly provided by Dr. H. Gronemeyer (IGBMC, Illkirch, France). W752G and W752R mutations were created by overlap extension PCR, and all $A R$ expression plasmids were sequenced. ARE(RAD9A) ${ }_{6}$-collagenase-luciferase-hygromycine was constructed by introduction of 6 copies of the ARE RAD9A (CCAAGGCTCTGGTAGTTCTTGGA) [Moehren et al., 2008] in front of the collagenase matrix metallopeptidase $(-42 /+48)$ promoter in the collagenase-luciferase-hygromycine plasmid. Transient transfections assays were performed in U2OS cells using Jet-PEI (Ozyme, Saint-Quentin en Yvelines, France) according to the manufacturer's instructions. Luciferase assays were performed with the Promega dual reporter kit according to the manufacturer's instructions. Renilla luciferase encoded by the normalization vector phRLTK (Promega) was used as an internal con- 
trol for firefly luciferase normalization. Tests were performed in duplicate in at least 3 independent experiments. Data were expressed as mean \pm SD of the percentage of luciferase expression, and $100 \%$ was the value obtained with AR-WT at $100 \mathrm{nM} \mathrm{R} 1881$.

Competitive Binding Assays

Briefly, U2OS cells were transiently transfected with AR-WT, AR-W752G, and AR-W752R. Expressing plasmids were seeded at a density of 50,000 cells per well in 96-well tissue culture plates and grown in DMEM supplemented with $5 \%$ dextran-coated, charcoal-treated FCS. After $20 \mathrm{~h}$, cells were labeled with increasing concentrations (1 pM-10 nM) of $\left[{ }^{3} \mathrm{H}\right]-\mathrm{R} 1881(72.6 \mathrm{Ci} / \mathrm{mmol}$ specific activity) at $37^{\circ} \mathrm{C}$ for $3 \mathrm{~h}$ in the absence or presence of unlabeled R1881 ( $1 \mu \mathrm{M})$. After incubation, unbound material was aspirated and the cells were washed 3 times with cold PBS. $\left[{ }^{3} \mathrm{H}\right]$-bound radioactivity was then counted by liquid scintillation. Nonspecific binding was determined in the presence of $1 \mu \mathrm{M}$ unlabeled R1881. Specific binding was calculated by subtracting nonspecific binding from total binding. Bound radioactivity was expressed in dpm (disintegrations per minute) per well. Tests were performed in quadruplicate in at least 3 independent experiments.

\section{Crystallographic Modeling}

Models of W752G or W752R mutant AR proteins were based on the structure of WT-AR (PDB code $3 \mathrm{~L} 3 \mathrm{X}$ ) and generated using WinCoot [Emsley and Cowtan, 2004].

\section{Results}

We identified an unreported c.2254T $>$ G, p.W752G mutation in exon 5 of the AR gene. The newborn's mother was found to be a heterozygous carrier for the mutation (Fig. 1). The mother's 46,XY sister refused molecular analysis of the $A R$ gene. As expected, the transfection studies confirmed the dramatic decrease in AR transactivation despite the increasing concentration of androgens in the culture medium. Conversely, for the other AR mutation (W752R) previously reported in 2 sisters with CAIS, the transactivation increased up to $50 \%$ of wild type when the androgen concentration was above $10^{-8} \mathrm{M}$ (Fig. 2). To determine whether the effects observed in the transactivation assays reflected a decrease in the binding affinity of androgens for AR mutants, $\left[{ }^{3} \mathrm{H}\right]-\mathrm{R} 1881$ "whole cell" assays were performed with U2OS cells transiently transfected with AR-WT and mutants-expressing plasmids. As expected, the AR mutants bind R1881 with lower affinity than AR-WT. AR-W752R displays a 100-fold lower affinity for R1881 than AR-WT, whereas no detectable binding activity was observed for AR-W752G, even at the highest $\left(10^{-8} \mathrm{M}\right)$ ligand concentration used (Fig. 3 ).

The structural analysis is presented in Figure 4 and shows that residue W752 is located in helix 5 (H5) (Fig. 4A), which is an important component of the hor-

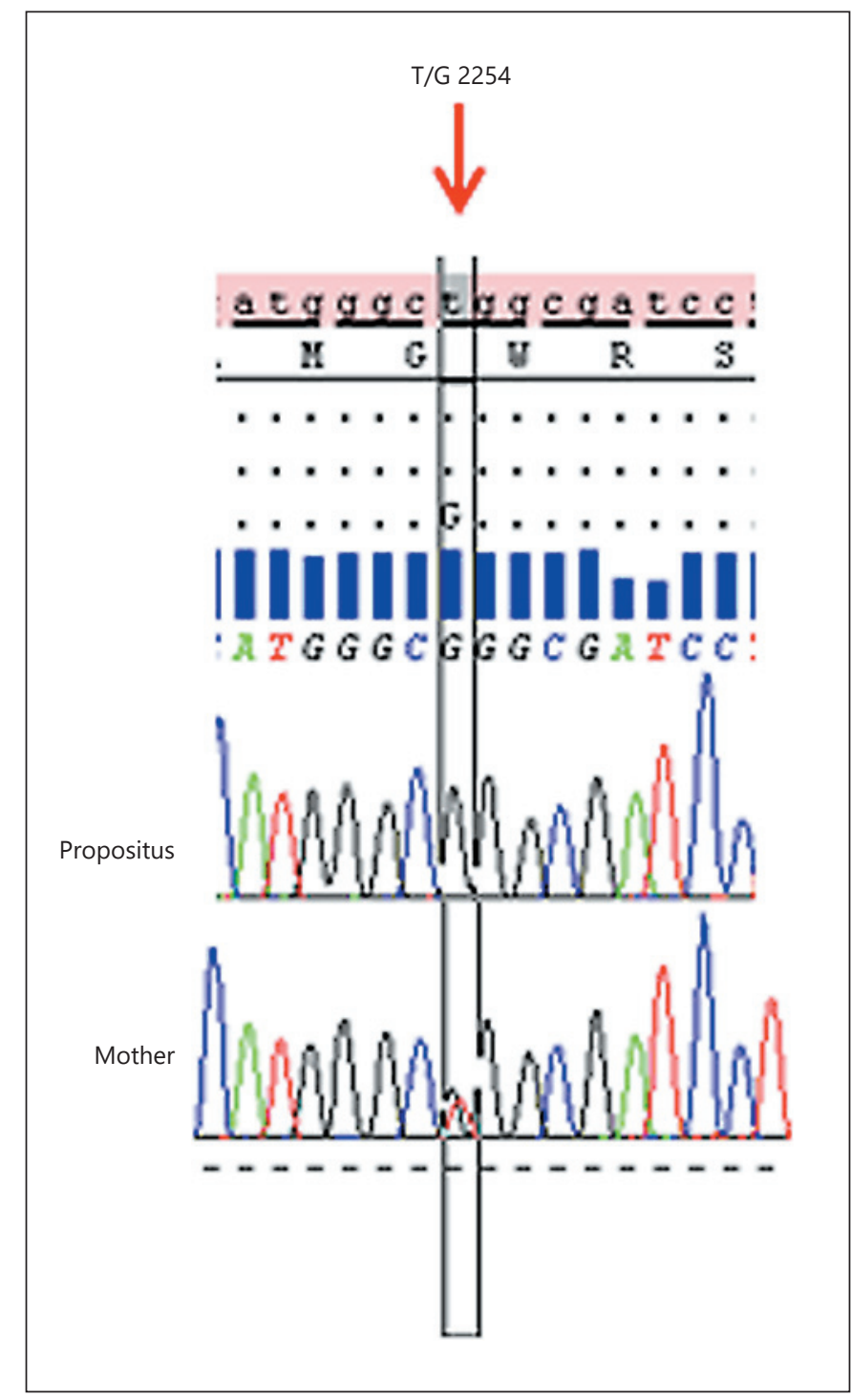

Fig. 1. $A R$ gene mutation, c. $2254 \mathrm{~T}>\mathrm{G}, \mathrm{p} . \mathrm{W} 752 \mathrm{G}$, in the propositus and the mother who is a heterozygous carrier for the mutation.

mone-binding pocket (HBP). The HBP of the AR is lined with 18 mostly hydrophobic residues that interact with the steroid scaffold of androgens like DHT. A few polar residues located at the 2 ends of the pocket form hydrogen bonds with the polar groups at the 3 and 17 positions of androgens. In particular, arginine 753 (R753) located in helix H5 contributes to a network of direct and watermediated hydrogen bonds that is critically involved in the stabilization of the hormone in the HBP (black dashed lines in Figure 4B). R753 plays a prominent role in the network as it (a) is directly linked to the 3-keto group of DHT, (b) stabilizes the hydrogen bond between Q712 
Fig. 2. U2OS cells transiently transfected with the reporter recombinant ARE (RAD9) ${ }_{6}$-collagenase-Luc-hygromycine and with or without pSG5-AR WT, -AR W752G, and -AR W752R and the reporter recombinant ARE(RAD9) 6 -collagenaseLuc-hygromycine. Transfected cells were incubated without and with and R1881 (3× $\left.10^{-11}-10^{-7} \mathrm{M}\right)$ to assess the potency of the different ARs. Luciferase assays were performed with the Promega dual reporter kit according to the manufacturer's instructions. Renilla luciferase encoded by the normalization vector phRLTK was used as internal control for firefly luciferase normalization. Data were expressed as mean \pm SD of the percentage of luciferase expression, and $100 \%$ was the value obtained with AR WT at 100 nM R1881.

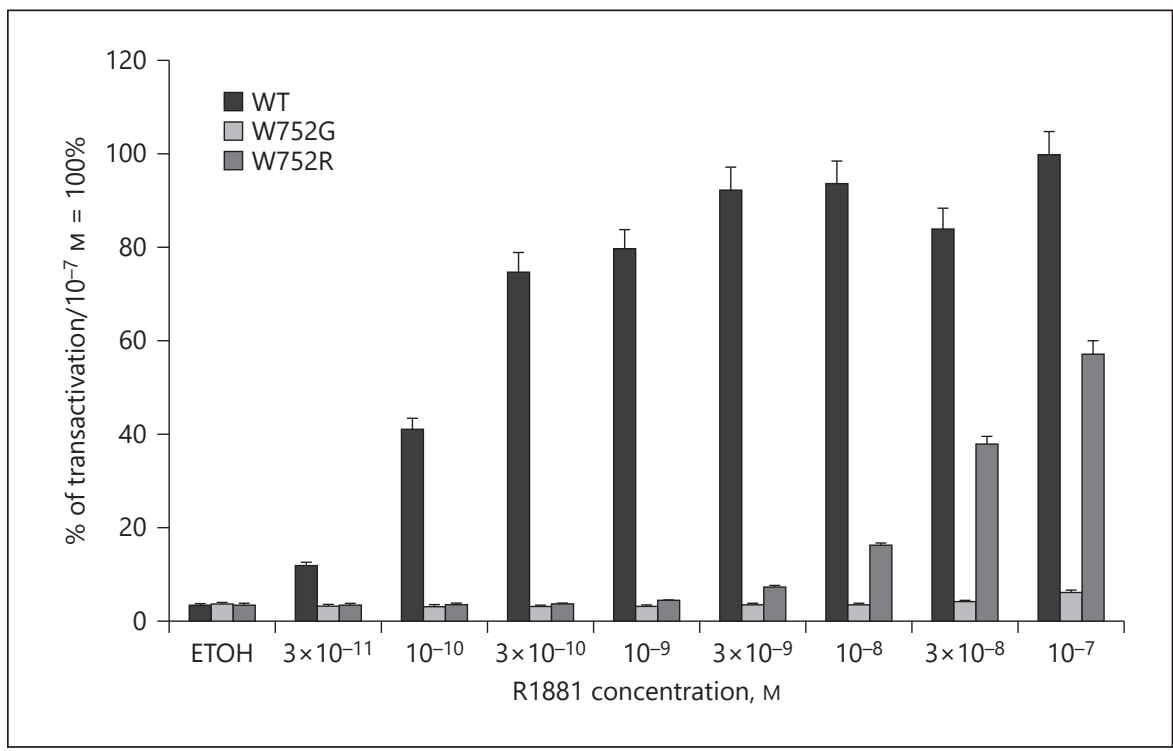

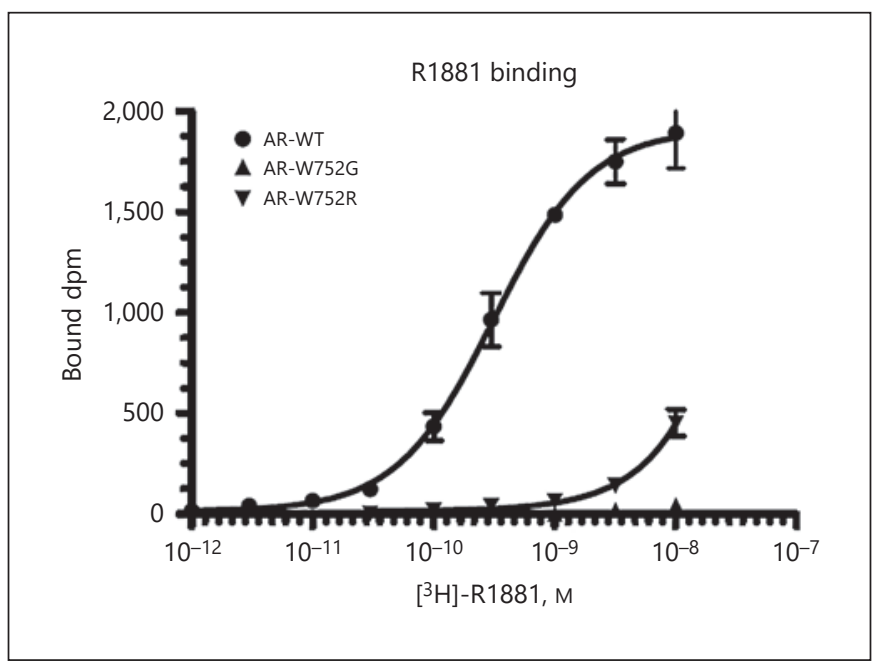

Fig. 3. Competitive binding assay. U2OS cells were transiently transfected with AR-WT, AR-W752G, and AR-W752R. Cells were labeled with increasing concentrations (1 pM to $10 \mathrm{nM})$ of $\left[{ }^{3} \mathrm{H}\right]$ $\mathrm{R} 1881$ (72.6 Ci/mmol specific activity) in the absence or presence of unlabeled R1881 (1 $\mu \mathrm{M})$. Bound radioactivity was expressed in dpm (disintegrations per minute) per well.

(helix H3) and DHT by maintaining the glutamine side chain in an optimal position via a water-mediated interaction, and (c) is hydrogen-bonded to Y764, thereby stabilizing the $\beta$-strand $S 1$ that contains residues in contact with the ligand. The mutation of W752 into a glycine residue (Fig. 4C) is very likely to have drastic structural ef- fects with significant functional consequences. As a strong $\alpha$-helix breaker, G752 will perturb the helical conformation of helix $\mathrm{H} 5$ and, as a consequence, it will destabilize the proper positioning of R753 and the hydrogen bond network anchoring the hormone (denoted by the absence of dashed lines in Figure 4C). In addition, the bulky side chain of W752 is involved in many van der Waals interactions with surrounding residues from helices $\mathrm{H} 7$ and $\mathrm{H} 8$ and the connecting loop (Fig. 4B). Because glycine residues do not contain any side chain, all the side chain-mediated interactions stabilizing the $\mathrm{H} 5 / \mathrm{H} 7 / \mathrm{H} 8$ region will be lost in the $\mathrm{W} 752 \mathrm{G}$ mutant. The residue R752 will have a much lower impact than G752 on the helical conformation of $\mathrm{H} 5$ and, even if some of them are lost, part of the interactions between the W752 side chain and the $\mathrm{H} 7$ and $\mathrm{H} 8$ residues can be reproduced by the R752 side chain.

\section{Discussion}

The clinical presentation of bilateral inguinal hernia in a newborn with female phenotype was classical. As Sultan et al. [2014] and Audi et al. [2010] reported, in the complete phenotype the first diagnosis in a family may be prenatal (4.3\%), but it is usually equally distributed between infancy owing to an inguinal hernia (47.8\%) and puberty through primary amenorrhea (45.7\%). Regarding phenotypic girls with inguinal hernia, $1 \%$ may present CAIS [Sarpel et al., 2005]. The current recommendation is to 


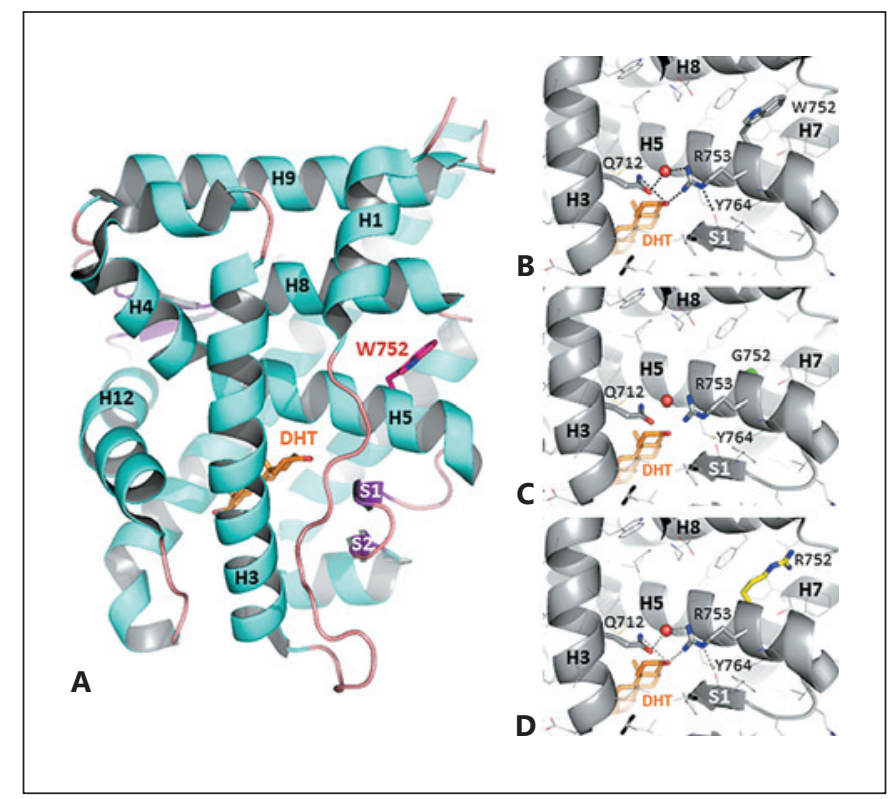

Fig. 4. Crystallographic modeling. A Crystal structure of the ligand binding domain of AR. The backbone of DHT is shown in orange with oxygen atoms shown in red. The side chain of residue W752 is displayed in red with the nitrogen atom in blue. Secondary structural elements are labeled ( $\mathrm{H}$, helix; $\mathrm{S}$, stand). B-D Close-up views on W752 and the amino acids it is mutated to (G or R). Side chains are shown and labeled. Selected amino acid side chains are shown, colored by atom type. Hydrogen bonds are indicated by dashed lines.

systematically consider karyotyping in all newborns with female phenotype and inguinal hernia to rule out CAIS [Deeb and Hughes, 2005; Georgiou et al., 2013].

Newborns diagnosed with CAIS are always raised as females, but the time of gonadectomy tends to be later than it was in the past years [Patel et al., 2016]. Later gonadectomy allows spontaneous puberty, but it nevertheless raises the question not only of increased testicular tumor risk [Dohnert et al., 2017] but also of both potential pubertal virilization and male identity in cases of persistent residual AR activity. A fundamental issue is thus to determine whether the type of mutation influences pubertal virilization through residual AR activity, which, if so, would argue for bringing forward the time of gonadectomy.

The mutation we identified in our patient was located in exon 5 of the $A R$ gene and caused an amino acid change p.W752G in the LBD of the AR. In 2 sisters with CAIS, a mutation located in the same position was previously described, changing the W752 to arginine [Brinkmann et al., 1995; Boehmer et al., 2001]. Nevertheless, a clear clin- ical presentation was not given at the age of diagnosis or regarding the clinical evolution with age. In addition, no functional assay was reported. To better hypothesize the future pubertal evolution based on the nature of the amino acid substitution, we performed an in vitro study of the new W752G substitution in parallel with the reported W752R mutation [Brinkmann et al., 1995; Boehmer et al., 2001]. Whereas the W752G mutation carried by our patient led to a collapse of AR transactivation whatever the androgen concentration, the previously reported W752R mutation presented some transactivational activity from a concentration of $10^{-8} \mathrm{M}$ to reach more than $50 \%$ of the wild-type AR transcriptional activity at $10^{-7} \mathrm{M}$, which is the range of androgen concentration found in the pubertal period.

The potential structural consequences of this mutation are found in the LBD, which is mapped to the $\mathrm{C}$ terminus and is composed of 12 a helices arranged in a globular structure, surrounding a central hydrophobic cavity or ligand-binding pocket. Ligand binding induces conformational changes, resulting in a profound positional shift of a helix 12 (H12) in the extreme C-terminal portion of the LBD. Ligand-induced movement of $\mathrm{H} 12$ is critical for co-regulator recruitment. As a consequence, ligand binding changes receptor interaction with co-regulators. The LBD involved in hormone binding belongs to the C-terminal part, which is also composed of the AF-2 domain, the second sequence responsible for the activation of target gene transcription and the region responsible for the dimerization of the receptor. The $\mathrm{N}$-terminal domain (A/B domains) and C-terminal domain (E domain) act as docking sites for transcriptional co-regulators [Helsen et al., 2012] and hence exhibit autonomous transactivation functions (AFs), designated as AF-1 and AF-2, respectively. AF-1 activity is ligand independent, whereas AF-2 is revealed upon ligand binding. A ligand-dependent functional interaction between the $\mathrm{N}$-terminal and $\mathrm{C}$-terminal regions suggests that AF- 1 and $\mathrm{AF}-2$ synergize to achieve full AR transactivation. The relative transactivation levels of AF-1 and AF-2 are variable and depend on the cellular environment, which presumably reflects the activity and expression levels of the relevant co-regulators [Rajender et al., 2008]. Moreover, post-translational modifications of the AR protein, such as phosphorylation or acetylation, also modulate the activities of AF-1 and AF-2.

Like other groups, we reported evidence that structural analysis has been proven useful to better understand the mechanism responsible for the alteration in AR transcriptional activity [Poujol et al., 2000, 2002; Georget et 
al., 2006]. The structural modifications induced by the W752G mutation very likely account for the complete androgen insensitivity of the AR-W752G mutant. Overall, compared with the $\mathrm{W}$ to $\mathrm{G}$ substitution, the structural perturbations induced by the W752R mutation were thus expected to affect the hormone-binding capacity of the receptor much less drastically (denoted by gray dashed lines in Figure 4D). In conclusion, our structural analysis is in line with the functional data reported here, showing residual transcriptional activity of $\mathrm{AR}$ at high pubertal androgen concentrations for AR-W752R; this was not found for AR-W752G, leading to greater alteration in AR transactivation.

Not only did the structural study provide a deeper understanding of the transcriptional AR activity based on the type of amino acid substitution, it also suggests that residual AR activity may be a risk for future pubertal virilization, which would justify the proposal of earlier gonadectomy. This risk has already been well described in PAIS patients raised as women, prompting consensus on early pubertal gonadectomy, especially given that there are more gonadal tumors in PAIS than in CAIS [Griffin, 1992; Mendoza and Motos, 2013]. Regarding CAIS, when the gonads are conserved until puberty, the risk of pubertal virilization should be kept in mind even though it is much lower than in PAIS patients raised as females [Cheikhelard et al., 2008]. Puberty occurs spontaneously in CAIS patients with maintained gonads, producing testosterone in the typical pubertal range that is aromatized into estrogens [Hughes and Deeb, 2006]. The risk of malignancy in CAIS before puberty and adolescence is generally low, with a range of $0.8-2 \%$ [Cools et al., 2006; Hughes and Deeb, 2006], but it increases with age. This risk does not seem to be linked to a type of mutation, although it appears to be higher in carriers of a LBD mutation [Gottlieb et al., 2004]. The recent trend is thus to delay gonadectomy until adolescence [Hughes and Deeb, 2006], which also allows patients to make their own management decision. This point should be emphasized because of the current ethical and legal problems raised by the report of spermatogonia in resected testis in several series [Rutgers and Scully, 1991; Cools et al., 2006; Hannema et al., 2006]. There are many concerns regarding early gonadectomy in CAIS patients, even in cases where in vitro confirmation of residual AR activity might require particular follow-up in the pubertal period.

We nevertheless propose that CAIS patients presenting residual AR activity at high $\mathrm{T}$ concentration should be more closely followed as puberty approaches so that gonadectomy can be advanced to avoid pubertal virilization which might induce gender identity dysphoria.

In conclusion, through the demonstration of differential transcriptional residual AR activity between our new W752G mutation of the $A R$ gene in a patient with CAIS and a previously reported W752R mutation in a newborn with the same phenotype, we hypothesize that there is a risk of pubertal virilization in case of residual AR transcriptional activity. We thus underline the usefulness of an in vitro study to better understand the virilization defect, predict the risk of pubertal virilization, and thus better organize patient follow-up and time of gonadectomy based on the nature of the amino acid substitution.

\section{Statement of Ethics}

The authors have no ethical conflicts to disclose.

\section{Disclosure Statement}

The authors have no conflicts of interest to declare.

\section{References}

Audi L, Fernandez-Cancio M, Carrascosa A, Andaluz P, Toran N, et al: Novel (60\%) and recurrent (40\%) androgen receptor gene mutations in a series of 59 patients with a $46, X Y$ disorder of sex development. J Clin Endocrinol Metab 95:1876-1888 (2010).

Boehmer AL, Brinkmann O, Bruggenwirth H, van Assendelft C, Otten BJ, et al: Genotype versus phenotype in families with androgen insensitivity syndrome. J Clin Endocrinol Metab 86:4151-4160 (2001).
Brinkmann AO, Jenster G, Ris-Stalpers C, van der Korput JA, Bruggenwirth HT, et al: Androgen receptor mutations. J Steroid Biochem Mol Biol 53:443-448 (1995).

Cheikhelard A, Morel Y, Thibaud E, Lortat-Jacob $S$, Jaubert F, et al: Long-term follow-up and comparison between genotype and phenotype in 29 cases of complete androgen insensitivity syndrome. J Urol 180:1496-1501 (2008).
Cools M, Drop SL, Wolffenbuttel KP, Oosterhuis JW, Looijenga LH: Germ cell tumors in the intersex gonad: old paths, new directions, moving frontiers. Endocr Rev 27:468-484 (2006).

Deeb A, Hughes IA: Inguinal hernia in female infants: a cue to check the sex chromosomes? BJU Int 96:401-403 (2005).

Dohnert U, Wunsch L, Hiort O: Gonadectomy in complete androgen insensitivity syndrome: why and when? Sex Dev 11:171-174 (2017). 
Eisermann K, Wang D, Jing Y, Pascal LE, Wang $\mathrm{Z}$ : Androgen receptor gene mutation, rearrangement, polymorphism. Transl Androl Urol 2:137-147 (2013).

Emsley P, Cowtan K: Coot: model-building tools for molecular graphics. Acta Crystallogr D Biol Crystallogr 60:2126-2132 (2004).

- Georget V, Bourguet W, Lumbroso S, Makni S, Sultan C, Nicolas JC: Glutamic acid 709 substitutions highlight the importance of the interaction between androgen receptor helices $\mathrm{H} 3$ and $\mathrm{H} 12$ for androgen and antiandrogen actions. Mol Endocrinol 20:724-734 (2006).

-Georgiou R, Hall NJ, Stanton M: Screening for complete androgen insensitivity syndrome in girls with inguinal hernia: parental insight. Arch Dis Child 98:316-317 (2013).

Gottlieb B, Beitel LK, Wu J, Elhaji YA, Trifiro M: Nuclear receptors and disease: androgen receptor. Essays Biochem 40:121-136 (2004).

-Gottlieb B, Beitel LK, Nadarajah A, Paliouras M, Trifiro M: The androgen receptor gene mutations database: 2012 update. Hum Mutat 33: 887-894 (2012).

Griffin JE: Androgen resistance-the clinical and molecular spectrum. N Engl J Med 326:611618 (1992).

-Hannema SE, Scott IS, Rajpert-De Meyts E, Skakkebaek NE, Coleman N, Hughes IA: Testicular development in the complete androgen insensitivity syndrome. J Pathol 208:518-527 (2006).
Helsen C, Dubois V, Verfaillie A, Young J, Trekels $\mathrm{M}$, et al: Evidence for DNA-binding domainligand-binding domain communications in the androgen receptor. Mol Cell Biol 32: 3033-3043 (2012).

Hughes IA, Deeb A: Androgen resistance. Best Pract Res Clin Endocrinol Metab 20:577-598 (2006).

Hurme T, Lahdes-Vasama T, Makela E, Iber T, Toppari J: Clinical findings in prepubertal girls with inguinal hernia with special reference to the diagnosis of androgen insensitivity syndrome. Scand J Urol Nephrol 43:42-46 (2009).

Mendoza N, Motos MA: Androgen insensitivity syndrome. Gynecol Endocrinol 29:1-5 (2013).

Moehren U, Denayer S, Podvinec M, Verrijdt G, Claessens F: Identification of androgen-selective androgen-response elements in the human aquaporin-5 and Rad 9 genes. Biochemical J 411:679-686 (2008).

Patel V, Casey RK, Gomez-Lobo V: Timing of gonadectomy in patients with complete androgen insensitivity syndrome - current recommendations and future directions. J Pediatr Adolesc Gynecol 29:320-325 (2016).

Philibert P, Audran F, Pienkowski C, Morange I, Kohler B, et al: Complete androgen insensitivity syndrome is frequently due to premature stop codons in exon 1 of the androgen receptor gene: an international collaborative report of 13 new mutations. Fertil Steril 94: 472-476 (2010).
Poujol N, Wurtz J, Tahiri B, Lumbroso S, Makni $S$, et al: Specific recognition of androgens by their nuclear receptor. A structure-function study. J Biol Chem 275:24022-24031 (2000).

- Poujol N, Lumbroso S, Makni S, Terouanne B, Lobaccaro J, et al: Pathophysiology of androgen insensitivity syndromes: molecular and structural approaches of natural and engineered androgen receptor mutations at amino acid 743. J Clin Endocrinol Metab 87: 5793-5800 (2002).

Rajender S, Gupta NJ, Chakravarty B, Singh L, Thangaraj K: Androgen insensitivity syndrome: do trinucleotide repeats in androgen receptor gene have any role? Asian J Androl 10:616-624 (2008).

Rutgers JL, Scully RE: The androgen insensitivity syndrome (testicular feminization): a clinicopathologic study of 43 cases. Int J Gynecol Pathol 10:126-144 (1991).

Sarpel U, Palmer SK, Dolgin SE: The incidence of complete androgen insensitivity in girls with inguinal hernias and assessment of screening by vaginal length measurement. J Pediatr Surg 40:133-136; discussion 136-137 (2005).

Sultan C, Philibert P, Gaspari L, Audran F, Maimoun L, et al: Chapter 5. Androgen insensitivity syndrome, in New MI, Lekarev O, Parsa A, Yuen TT, O'Malley B, Hammer GD (eds): Genetic Steroid Disorders, pp 225-237 (Academic Press, Cambridge 2014). 